Article

\title{
Empirical Prediction of Turnovers in NFL Football
}

\author{
Joel R. Bock
}

PointPredictive Inc., San Diego, CA 92120, USA; sauerkraut@gmail.com

Academic Editor: Eling Douwe de Bruin

Received: 18 October 2016; Accepted: 20 December 2016; Published: 29 December 2016

\begin{abstract}
Turnovers in the National Football League (NFL) occur whenever a team loses possession of the ball due to a fumble, or an interception. Turnovers disrupt momentum of the offensive team, and represent lost opportunities to advance downfield and score. Teams with a positive differential turnover margin in a given game win $70 \%$ of the time. Turnovers are statistically rare events, occurring apparently randomly. These characteristics make them difficult to predict. This investigation advances the hypothesis that turnovers are predictable in NFL football. Machine learning models are developed to learn the concept: At any point within a football game, what is the likelihood that a turnover will be observed on the next play from scrimmage? NFL play-by-play data for 32 teams spanning seven full seasons were used to train the models. Results presented suggest evidence to support the working hypothesis. Under certain conditions, both fumbles and interceptions can be anticipated at low false discovery rates (less than $15 \%$ ). When a turnover is predicted on the impending play from scrimmage, a high degree of confidence is associated with that prediction. The ability to anticipate catastrophic in-game events may lead to their management and control, ultimately improving the performance of individual athletes and their teams. This investigation contributes to the sports science literature by demonstrating the predictability of in-game events often considered to be essentially random in their occurrence. To the author's knowledge, direct prediction of turnovers has not previously appeared in the literature, which has focused on retrospective statistical analyses of turnover margin in football games.
\end{abstract}

Keywords: machine learning; unbalanced data; predicting rare events; NFL football; sports analytics; performance optimization

\section{Introduction}

Turnovers in the National Football League (NFL) occur whenever a team loses possession of the ball due to a fumble, or an interception. A fumble is any act other than passing, handoffs, or legally kicking the ball, which results in a loss of possession from offense to defense. Interceptions occur when a defender catches a pass or fumble that does not touch the ground [1]. Turnovers disrupt the momentum of the offensive team, and represent lost opportunities to advance downfield and score.

A positive differential turnover margin in a given game is a significant predictor correlated with winning that game [2]. One analysis of multi-season game outcomes found that teams having a single turnover less than their opponent were victorious $70 \%$ of the time [3].

Turnovers may be caused by any number of factors, including physical collisions, mistakes in play execution or lapses in player concentration. In statistical terms, turnovers are rare events. Less than $3 \%$ of passes are intercepted, and less than $1 \%$ of run plays end in fumbles being recovered by the defensive team. Turnovers appear to be random events [4]; previous work concluded that roughly equal parts chance and talent accounted for year-over-year variations in turnover differential for a given team [3]. Within a season, NFL turnovers are only weakly correlated with prior game performance [5]. 
These characteristics-rarity, irregular but recurrent appearance, and costliness in their effect-suggest a connection with so-called "extreme" events in dynamical systems, which are inherently difficult to predict [6].

The ability to anticipate turnovers with some degree of statistical confidence potentially offers significant value for in-game strategies to mitigate their negative consequences.

This investigation tests the hypothesis that turnovers are predictable in NFL football. Statistical models that predict the likelihood of observing a turnover on a given play from scrimmage are developed, and evaluated using play-by-play data from seven NFL seasons.

\section{Methods}

\subsection{Gradient Boosted Machine Learning}

The concept to be learned in this investigation is this: At any point within a football game, what is the likelihood that a turnover will be observed on the next play from scrimmage?

The specific objective is to learn an unknown function $F$ that maps explanatory variables $\mathbf{x}=\left\{x_{1}, \ldots, x_{d}\right\}$ to the response $y$, or $F: \mathbf{x} \rightarrow y$, where $\mathbf{x}$ represents the game situation, and $y \in\{0,1\}$ is the binary decision (no turnover, turnover). A collection of training examples $T=\left\{\left(\mathbf{x}_{i}, y_{i}\right), i=1, \ldots N\right\}$ is used to estimate an approximation to $F$, or $\hat{F}(\mathbf{x})=y$, by an adaptive learning algorithm known as the "gradient boosting machine" [7,8].

Gradient boosting machines (GBMs) are learning algorithms that reconstruct a decision function $\hat{F}$ based on the consensus of an ensemble of classification or regression trees. New decision tree models are sequentially added to the ensemble, in order to increase the estimation accuracy of the response variable. The numerical optimization minimizes an expected loss $\hat{F}(\mathbf{x})=\operatorname{argmin}_{F} E_{y, \mathbf{x}} L(y, F(\mathbf{x}))$ of a group of trees, conditioned over the entire training data set [7]. The loss function can be selected according to a given learning concept and joint probability distribution $f(\mathbf{x}, y)$ under study. Here, we use a Bernoulli distribution loss function, convert the classification to a continuous value via logistic regression and estimate the turnover probability $\hat{p}\left(\mathbf{x}_{\mathbf{i}}\right)=p\left(y_{i}=1 \mid \mathbf{x}_{\mathbf{i}}\right), \hat{p} \in[0,1]$.

A useful property of GBMs in applications is interpretability through calculation of the relative influence of explanatory variables in constructing a consensus decision. The influence of each input variable $x_{j}$ in a given tree is based on the frequency of its selection for splitting in non-terminal nodes, and its contribution to successful model classification over the data sample. This influence is averaged over the ensemble of trees to estimate the variable's overall importance to the decision function $\hat{F}$ [7]. In the current investigation, this interpretation may provide insight into the game conditions under which turnovers might be expected to occur.

Gradient boosting machine models were developed and evaluated in $R$, using the "gbm" package $[9,10]$.

\subsection{Sample, Segmentation and Features}

The population under study consists of NFL season, game, player and play-level data for complete seasons 2009 through 2015, covering all 32 teams. Game data were downloaded from the site www.nfl.com using utilities provided by "nflscrapR" [11]. These data were preprocessed by (1) sampling by season and team; (2) filtering by play type, to include only plays from scrimmage (run, pass or sack); (3) annotating by status of turnovers (true, false) observed on each play; (4) constructing feature vectors using attributes of the play-by-play and game contextual data.

This sample comprised 300,450 plays. Running plays represented $31.7 \%$ of all plays, passes $42.1 \%$, and sacks only $2.9 \%$. Although sack-fumbles lost are significant events $(5.1 \%$ of sacks produce turnovers), we decided to exclude sacks from further consideration due to their negligible numbers relative to run and pass plays. To make this predictive analysis useful in practice, it is prudent to categorize turnover events in association with scrimmage plays that could reasonably be anticipated by a defensive team, based on offensive formation. 
After excluding sack plays, the sample contained 291,675 plays, with an overall turnover prevalence of $1.633 \%$ for pass and run plays, combined. Pass plays made up $43.4 \%$ and runs $32.6 \%$ of the resultant dataset.

Two partitioning schemes were applied to the sample. First, an aggregate sample of all $32 \mathrm{NFL}$ teams was created to assess whether invariant patterns of turnover predictability could be determined. Second, individual team samples were assembled to develop team-specific models of turnovers. Seven full season-long records were used for all sample datasets.

Predictive models were trained and evaluated for each sample. These samples were segmented by distinct event types-(1) Run plays; (2) Pass plays; and (3) Run or Pass plays combined.

Feature vectors for learning were constructed from available fields in the play-by-play data. Numeric data were normalized by characteristic length and time scales. Categorical and ordinal variables were represented as binary valued quantities using one-hot encoding. The features and their corresponding nominal dimensions upon encoding are summarized in Table 1. Not all dimensions listed in the table were in model development, due to their low variation across certain limited subsamples.

Table 1. Features used in models to predict National Football League (NFL) turnovers.

\begin{tabular}{cc}
\hline Feature & Dimension \\
\hline Drive number & 1 \\
Quarter & 5 \\
Down & 4 \\
Remaining game time & 1 \\
Yard line & 1 \\
Yards to 1st down & 1 \\
Yards on drive, net & 1 \\
Play type & 14 \\
Score differential & 1 \\
Run location & 3 \\
Pass location & 3 \\
Last run location & 3 \\
Last pass location & 3 \\
Last pass outcome & 3 \\
Last play yards gained & 3 \\
\hline
\end{tabular}

\subsection{Modeling and Analysis}

The incidence of turnovers as a percentage of all plays from scrimmage is very low, around $1.6 \%$. For this reason, the distribution of class labels $y_{i}$ in a training set $T=\left\{\left(\mathbf{x}_{i}, y_{i}\right), i=1, \ldots N\right\}$ randomly sampled from the true population is highly skewed. Learning the parameters of a useful statistical estimator of turnover probability $\hat{p}\left(\mathbf{x}_{\mathbf{i}}\right)=p\left(y_{i}=1 \mid \mathbf{x}_{\mathbf{i}}\right)$ suggests the use of specific learning techniques to avoid trivially predicting "no turnover" on every decision [12].

To address this, the approach taken in this study was to re-balance the distribution of classes in the training set, over-representing the distribution of the minority class in order to present sufficient examples to the learning algorithm. During validation of the models, examples closely representative of the true distribution within the population were used to assess model predictive power when applied out-of-sample.

The modeling strategy included bootstrap resampling [13], cross validation analysis, and receiver operating characteristic curve (ROC) analysis [14]. The latter technique enabled error estimation, model comparison and selection from the large number of hypotheses generated by the gradient boosting machines during training. ROC curves are often used to tradeoff false positive rate (FPR) and true positive rate $(T P R)$ for evaluation of classifiers. In this study, the false discovery rate $(F D R)$ was substituted for $F P R$ for analysis. FDR is the fraction of all positive decisions (i.e., turnover predicted) made by a model that are incorrect [15]. FDR is a more informative metric than FPR in diagnostic or predictive applications where confidence in a positive prediction is preferred, especially when the class 
distribution is skewed [16]. FDR is related to the positive predictive value statistic by $P P V=1-F D R$. High PPV (low FDR) values are desirable. TPR denotes the sensitivity of the model, or the likelihood that actual turnovers events are detected within a testing distribution.

In ROC space ( $F D R, T P R)$, an optimal decision threshold $D T_{\text {opt }}$ is determined experimentally for a given distribution. Our objective is to minimize $F D R$ for tactical reasons. A second pass through the training data with this fixed threshold is used to train and evaluate model performance. The gradient boosted model outputs a probability $\hat{p}$; the turnover prediction algorithm is then [17]

$$
\hat{y}(\mathbf{x})= \begin{cases}1: & \text { if } \hat{p}>D T_{\text {opt }} \\ 0: & \text { if } \hat{p} \leq D T_{\text {opt }}\end{cases}
$$

where $\hat{y}(\mathbf{x})=1$ means a turnover will be observed given input $\mathbf{x}$.

Model learning for the aggregate sample used the "bootstrap" [13] to repeatedly draw samples from the entire training set. Data were partitioned according to the play type segment under consideration, and a stratified sample was constructed for training. The validation data were sampled at random from the entire sample, according to the natural distribution of turnovers. A two step procedure was followed, for each of $B=100$ bootstrap replicates. The first step estimated the detection threshold (DT) for optimal FDR and TPR via ROC analysis, training GBMs comprising 1500 trees (nominally). Secondly, the threshold was held constant such that $D T=D T_{o p t}$ and the entire sample was modeled again.

The learning procedure used for the team-wise samples was notionally similar, differing slightly in the numerical mechanics. Stratified sampling (with respect to the class labels $y$ ) of individual teams produced untenably small sample counts. This required an alternate sampling strategy. The decision was made to use 10 -fold cross validation, nested within a 10-trial bagging procedure. Prediction rules were developed by finally averaging the performance results. Modeling therefore included all of the available instances, and benefited from the variance-reduction properties of bagging for model performance estimation [18].

Performance statistics $F D R\left(D T_{\text {opt }}\right), T P R\left(D T_{\text {opt }}\right)$ were accumulated and finally averaged over replicates $B$ (or trials/folds $k$ ) to estimate the generalization performance of the ensemble of trees. Sampling distributions of the sample mean and standard error values for FDR and TPR observed in out-of-sample test were recorded for each sample and segment under investigation.

In this investigation, we define a "good" false discovery rate to be $F D R<0.15$. In other words, a positive prediction made by the model $(\hat{y}(\mathbf{x})=1)$ is correct at least $85 \%$ of the time to meet this criterion of model utility. This means that when a turnover is predicted on the impending play from scrimmage, a high degree of confidence can be associated with that prediction.

A pseudo-code outline of the model training and evaluation procedure appears in the Appendix, as Algorithm A1.

\section{Results}

The main predictive modeling results of this paper are presented in Tables 2-5. These tables list the sampling distributions of the sample mean and standard errors for observed false discovery (FDR) and true positive rates (TPR) on predicting turnovers for the sample data segments and event types considered. These are the primary statistics used to evaluate turnover prediction acuity. The results tables also include observed values for prevalence of actual turnovers (Prev.) and corresponding out-of-sample instance counts $\left(N_{\text {oos }}\right)$.

We define a "good" false discovery rate to be $F D R<0.15$. Tabulated results are annotated in green to highlight predictions meeting or exceeding this threshold for low FDR. True positive rates observed, where TPR $>0.60$, are similarly noted in the tables.

Team-aggregated performance results are found in Table 2. Model predictions by individual team and segment appear in Tables 3-5 . 
A synopsis of the important input variables contributing to the ensemble prediction for one of the samples studied is presented in Table 6 .

\subsection{Turnover Prediction Results-Aggregated}

Table 2 summarizes performance of models for the aggregate sample, for each of the three turnover event types considered. Low values of FDR are seen for each segment $(6.4 \%, 8.3 \%$, and $3.9 \%$ for Run or Pass, Pass and Run, respectively). For Run plays, the sensitivity TPR is seen to be 0.65 , making this segment the most successfully predicted turnover event type in the sample.

The results shown in Table 2 lend support to our hypothesis that turnovers are predictable in NFL football. Given an educated guess as to the expected play type from scrimmage, the GBM models have excellent positive predictive value in estimating turnover likelihood.

Table 2. Aggregate prediction performance: false discovery rate $(F D R)$ and true positive rate $(T P R)$ of models. A total of 32 teams, seasons 2009-2015. $B=100$.

\begin{tabular}{ccccc}
\hline Segment & FDR $( \pm \mathbf{s e})$ & TPR $( \pm$ se $)$ & Prev. & $\boldsymbol{N}_{\text {oos }}^{B}$ \\
\hline Run or Pass & $0.064(0.026)$ & $0.417(0.023)$ & 0.0164 & 22,183 \\
Pass & $0.083(0.031)$ & $0.381(0.028)$ & 0.024 & 12,653 \\
Run & $0.039(0.035)$ & $0.650(0.064)$ & 0.006 & 9514 \\
\hline
\end{tabular}

\subsection{Turnover Prediction Results_-Team-Wise}

Prediction results for team-wise samples of the Run or Pass segment are found in Table 3. These statistics show that 20 of the 32 team models (62.5\%) are observed to have FDR rates below the 0.15 goodness criterion as defined here. The best performing team model in terms of false discovery rate was for the New England Patriots (NE), where the sample mean FDR was 2.1\%. Cleveland (CLE), Buffalo (BUF), San Diego (SD), Minnesota (MIN), Tennessee (TEN) and Seattle (SEA) each had FDR's of less than $10 \%$. San Francisco (SF) produced the most frequent rate of false positives, at $26.9 \%$.

Table 3. Team-wise prediction performance, Run or Pass segment, seasons 2009-2015. $k=10$.

\begin{tabular}{ccccc}
\hline Team & FDR $( \pm$ se $)$ & TPR $( \pm$ se $)$ & Prev. & $N_{\text {oos }}^{k}$ \\
\hline NE & $0.021(0.073)$ & $0.449(0.112)$ & 0.008 & 6545 \\
CLE & $0.087(0.109)$ & $0.467(0.133)$ & 0.018 & 6117 \\
CHI & $0.134(0.124)$ & $0.359(0.125)$ & 0.019 & 5974 \\
HOU & $0.177(0.168)$ & $0.453(0.138)$ & 0.014 & 6475 \\
NYJ & $0.193(0.162)$ & $0.431(0.105)$ & 0.019 & 6378 \\
BUF & $0.097(0.097)$ & $0.461(0.151)$ & 0.020 & 6072 \\
WAS & $0.117(0.138)$ & $0.420(0.097)$ & 0.017 & 6161 \\
JAC & $0.132(0.116)$ & $0.429(0.118)$ & 0.018 & 6042 \\
STL & $0.146(0.110)$ & $0.531(0.108)$ & 0.016 & 5527 \\
ARI & $0.135(0.119)$ & $0.408(0.120)$ & 0.020 & 6081 \\
SD & $0.063(0.111)$ & $0.493(0.074)$ & 0.014 & 6222 \\
TB & $0.164(0.124)$ & $0.472(0.113)$ & 0.019 & 5940 \\
OAK & $0.113(0.120)$ & $0.453(0.146)$ & 0.019 & 6112 \\
DEN & $0.174(0.126)$ & $0.491(0.143)$ & 0.015 & 6454 \\
DAL & $0.180(0.151)$ & $0.412(0.112)$ & 0.015 & 6108 \\
ATL & $0.177(0.171)$ & $0.433(0.139)$ & 0.015 & 6403 \\
SF & $0.269(0.194)$ & $0.446(0.208)$ & 0.010 & 5802 \\
KC & $0.195(0.194)$ & $0.539(0.203)$ & 0.014 & 6073 \\
CAR & $0.189(0.147)$ & $0.425(0.134)$ & 0.016 & 6164 \\
PIT & $0.116(0.124)$ & $0.498(0.125)$ & 0.016 & 6111 \\
NO & $0.189(0.208)$ & $0.463(0.141)$ & 0.015 & 6584 \\
MIN & $0.068(0.110)$ & $0.451(0.086)$ & 0.016 & 6031 \\
CIN & $0.128(0.113)$ & $0.507(0.141)$ & 0.018 & 6272 \\
NYG & $0.103(0.092)$ & $0.480(0.147)$ & 0.021 & 6291 \\
PHI & $0.124(0.110)$ & $0.478(0.131)$ & 0.019 & 6398 \\
GB & $0.188(0.185)$ & $0.485(0.176)$ & 0.010 & 6142 \\
IND & $0.140(0.131)$ & $0.431(0.125)$ & 0.017 & 6360 \\
\hline & & & &
\end{tabular}


Table 3. Cont.

\begin{tabular}{ccccc}
\hline Team & FDR $( \pm$ se $)$ & TPR $( \pm$ se $)$ & Prev. & $N_{\text {oos }}^{k}$ \\
\hline TEN & $0.091(0.102)$ & $0.473(0.112)$ & 0.020 & 5876 \\
BAL & $0.126(0.116)$ & $0.531(0.102)$ & 0.015 & 6321 \\
MIA & $0.113(0.126)$ & $0.493(0.121)$ & 0.017 & 6089 \\
SEA & $0.097(0.171)$ & $0.551(0.191)$ & 0.013 & 6000 \\
DET & $0.113(0.121)$ & $0.452(0.075)$ & 0.019 & 6574 \\
\hline
\end{tabular}

Team abbreviations: NE: New England Patriots; CLE: Cleveland Browns; CHI: Chicago Bears; HOU: Houston Texans; NYJ: New York Jets; BUF: Buffalo Bills; WAS: Washington Redskins; JAC: Jacksonville Jaguars; STL: St. Louis Rams; ARI: Arizona Cardinals; SD: San Diego Chargers; TB: Tampa Bay Buccaneers; OAK: Oakland Raiders; DEN: Denver Broncos; DAL: Dallas Cowboys; ATL: Atlanta Falcons; SF: San Francisco 49ers; KC: Kansas City Chiefs; CAR: Carolina Panthers; PIT: Pittsburgh Steelers; NO: New Orleans Saints; MIN: Minnesota Vikings; CIN: Cincinnati Bengals; NYG: New York Giants; PHI: Philadelphia Eagles; GB: Green Bay Packers; IND: Indianapolis Colts; TEN: Tennessee Titans; BAL: Baltimore Ravens; MIA: Miami Dolphins; SEA: Seattle Seahawks; DET: Detroit Lions.

Note that the standard errors of the sample mean of the $F D R$ statistic are a significant proportion of the sample mean value. This behavior is seen in nearly all of the turnover prediction results obtained in this study. Model sensitivity TPR is moderate in general, ranging from $40 \%$ to $50 \%$. This statistic is considered less vital here than FDR, but is still important.

Details of modeling performance for Pass plays from scrimmage are presented in Table 4. For this segment, 10 of 32 teams (32\%) were associated with good turnover predictability as measured by FDR. The best precision was observed for Tennessee, where $F D R=0.077$. New England had the highest false positive rate at $30 \%$. The true positive rate of the method is seen to be generally low, in the $30 \%$ to low $40 \%$ range.

Table 4. Team-wise prediction performance, Pass segment, seasons 2009-2015. $k=10$.

\begin{tabular}{ccccc}
\hline Team & FDR $( \pm$ se $)$ & TPR $( \pm$ se $)$ & Prev. & $N_{\text {oos }}^{k}$ \\
\hline NE & $0.305(0.222)$ & $0.469(0.168)$ & 0.011 & 3807 \\
CLE & $0.1190 .115)$ & $0.445(0.146)$ & 0.026 & 3527 \\
CHI & $0.191(0.147)$ & $0.385(0.134)$ & 0.030 & 3404 \\
HOU & $0.174(0.183)$ & $0.376(0.155)$ & 0.020 & 3558 \\
NYJ & $0.205(0.170)$ & $0.339(0.109)$ & 0.032 & 3254 \\
BUF & $0.169(0.146)$ & $0.427(0.176)$ & 0.032 & 3327 \\
WAS & $0.229(0.162)$ & $0.415(0.101)$ & 0.026 & 3561 \\
JAC & $0.156(0.134)$ & $0.409(0.138)$ & 0.027 & 3485 \\
STL & $0.183(0.189)$ & $0.430(0.165)$ & 0.022 & 3400 \\
ARI & $0.134(0.137)$ & $0.400(0.134)$ & 0.028 & 3682 \\
SD & $0.120(0.159)$ & $0.447(0.097)$ & 0.021 & 3605 \\
TB & $0.204(0.154)$ & $0.449(0.129)$ & 0.027 & 3434 \\
OAK & $0.184(0.168)$ & $0.429(0.143)$ & 0.027 & 3535 \\
DEN & $0.149(0.180)$ & $0.428(0.177)$ & 0.022 & 3685 \\
DAL & $0.177(0.161)$ & $0.411(0.111)$ & 0.024 & 3603 \\
ATL & $0.176(0.173)$ & $0.434(0.131)$ & 0.021 & 3877 \\
SF & $0.269(0.288)$ & $0.345(0.222)$ & 0.016 & 3035 \\
KC & $0.196(0.208)$ & $0.433(0.241$ & 0.021 & 3183 \\
CAR & $0.246(0.232)$ & $0.377(0.151)$ & 0.026 & 3172 \\
PIT & $0.137(0.139)$ & $0.384(0.130)$ & 0.022 & 3545 \\
NO & $0.179(0.192)$ & $0.431(0.157)$ & 0.021 & 4119 \\
MIN & $0.198(0.182)$ & $0.420(0.109)$ & 0.025 & 3269 \\
CIN & $0.116(0.122)$ & $0.439(0.150)$ & 0.029 & 3427 \\
NYG & $0.164(0.153)$ & $0.378(0.151)$ & 0.029 & 3679 \\
PHI & $0.178(0.138)$ & $0.415(0.117)$ & 0.027 & 3698 \\
GB & $0.129(0.161)$ & $0.507(0.190)$ & 0.014 & 3504 \\
IND & $0.187(0.156)$ & $0.395(0.118)$ & 0.024 & 3936 \\
TEN & $0.077(0.099)$ & $0.455(0.104)$ & 0.029 & 3362 \\
BAL & $0.162(0.150)$ & $0.480(0.104)$ & 0.021 & 3599 \\
MIA & $0.150(0.175)$ & $0.408(0.149)$ & 0.024 & 3510 \\
SEA & $0.146(0.204)$ & $0.540(0.206)$ & 0.021 & 3127 \\
DET & $0.118(0.116)$ & $0.393(0.080)$ & 0.026 & 4133 \\
\hline & & & &
\end{tabular}


Large standard errors of the predicted sample mean FDR are apparent in these results.

Statistical performance of models trained on Run segment data is compiled in Table 5 . These models are seen to be less precise than observed for other segments, as 7 of 32 team models produced sample mean $F D R^{\prime}$ s of less than $15 \%$. It is clear that the overall sensitivity of the Run turnover predictions is very good, as $69 \%$ of the models (22/32) show true positive rates exceeding $60 \%$. This observation is consistent with the "rolled-up" results shown in Table 2, where the overall Run segment produces TPR $=0.65$.

It is interesting to note that the lowest turnover prevalence by play type in the population (for run plays) is associated with the highest degree of model sensitivity in out-of-sample predictions. The disparity in realized false positive rates between Tables 2 and 5 could be, in part, explained by the absolute number of training observations available in the aggregate case versus the team-wise samples, where fewer examples in the latter may be insufficient to learn the joint distribution between input and output variables.

Table 5. Team-wise prediction performance, Run segment, seasons 2009-2015. $k=10$.

\begin{tabular}{ccccc}
\hline Team & FDR $( \pm$ se $)$ & TPR ( \pm se $)$ & Prev. & $N_{\text {oos }}^{k}$ \\
\hline NE & $0.945(0.151)$ & $0.119(0.321)$ & 0.003 & 2537 \\
CLE & $0.230(0.325)$ & $0.657(0.429)$ & 0.006 & 2589 \\
CHI & $0.918(0.195)$ & $0.181(0.375)$ & 0.004 & 2569 \\
HOU & $0.196(0.336)$ & $0.762(0.362)$ & 0.006 & 2917 \\
\hline NYJ & $0.119(0.209)$ & $0.778(0.441)$ & 0.006 & 2895 \\
BUF & $0.500(0.343)$ & $0.683(0.404)$ & 0.006 & 2744 \\
WAS & $0.335(0.454)$ & $0.236(0.319)$ & 0.005 & 2600 \\
JAC & $0.777(0.287)$ & $0.463(0.442)$ & 0.005 & 2557 \\
STL & $0.106(0.195)$ & $0.901(0.161)$ & 0.007 & 2565 \\
ARI & $0.571(0.362)$ & $0.591(0.378)$ & 0.008 & 2351 \\
\hline SD & $0.143(0.339)$ & $0.665(0.344)$ & 0.005 & 2617 \\
TB & $0.317(0.373)$ & $0.660(0.400)$ & 0.008 & 2506 \\
OAK & $0.306(0.353)$ & $0.668(0.394)$ & 0.007 & 2577 \\
DEN & $0.389(0.332)$ & $0.603(0.362)$ & 0.007 & 2769 \\
DAL & $0.107(0.289)$ & $0.138(0.328)$ & 0.004 & 2505 \\
ATL & $0.337(0.353)$ & $0.293(0.230)$ & 0.005 & 2527 \\
SF & $0.550(0.415)$ & $0.728(0.405)$ & 0.004 & 2721 \\
KC & $0.279(0.370)$ & $0.827(0.248)$ & 0.007 & 2890 \\
\hline CAR & $0.266(0.278)$ & $0.707(0.326)$ & 0.005 & 2943 \\
PIT & $0.285(0.348)$ & $0.913(0.219)$ & 0.008 & 2566 \\
NO & $0.937(0.111)$ & $0.321(0.417)$ & 0.004 & 2465 \\
MIN & $0.182(0.274)$ & $0.733(0.244)$ & 0.006 & 2761 \\
CIN & $0.085(0.170)$ & $0.770(0.329)$ & 0.006 & 2844 \\
NYG & $0.131(0.185)$ & $0.867(0.201)$ & 0.010 & 2611 \\
PHI & $0.357(0.326)$ & $0.642(0.433)$ & 0.009 & 2700 \\
GB & $0.981(0.055)$ & $0.119(0.267)$ & 0.004 & 2638 \\
IND & $0.224(0.389)$ & $0.711(0.392)$ & 0.007 & 2424 \\
TEN & $0.155(0.218)$ & $0.650(0.255)$ & 0.009 & 2514 \\
BAL & $0.177(0.266)$ & $0.858(0.271)$ & 0.006 & 2722 \\
MIA & $0.274(0.318)$ & $0.807(0.331)$ & 0.007 & 2578 \\
SEA & $0.658(0.365)$ & $0.302(0.400)$ & 0.004 & 2874 \\
DET & $0.126(0.202)$ & $0.750(0.253)$ & 0.008 & 2441 \\
\hline & & & & \\
\hline
\end{tabular}

\subsection{Variable Influence}

The relative importance of variables in the feature vector is estimated by gradient boosted machines [7]. This estimate is based on the increase in log likelihood of a decision tree as determined by non-terminal splits made on each variable; this likelihood improvement is summed over all trees in the ensemble to apportion that variable's contribution to the plurality decision. 
The most and least influential variables for turnover predictions made for the aggregate sample are summarized in Table 6. The most important features are last_pass_complete and last_pass_incomplete. These are boolean valued features describing the outcome of the last pass from scrimmage. The remaining highly influential variables make intuitive sense, describing the current game situation (time remaining, score differential, yards to go for a first down or score). Two seemingly trivial variables (is_pass and is_run) are consistent with the composition of the Run or Pass segmentation, and strongly influence the splitting pattern of the constituent trees.

Non-important variables include the quarter of the game (subsumed by time_left), the direction of a current run play, and plays immediately following special teams activity.

Table 6. Most and least influential variables. Aggregate sample, Run or Pass segment.

\begin{tabular}{cccc}
\hline \multicolumn{2}{c}{ (a) Most Influential } & \multicolumn{2}{c}{ (b) Least Influential } \\
\hline Variable & Avg. Rank & Variable & Avg. Rank \\
\hline last_pass_complete & 1 & qtr2 & 27 \\
last_pass_incomplete & 2 & qtr4 & 28 \\
is_last_pass & 3 & is_last_punt & 29 \\
time_left & 4 & is_run_left & 30 \\
last_yards_gained & 5 & qtr1 & 31 \\
dwn1 & 6 & is_run_right & 32 \\
yds_drive & 7 & is_run_middle & 33 \\
is_run & 8 & is_last_ex_pt & 34 \\
is_pass & 9 & is_ot & 35 \\
score_diff & 10 & is_last_fld_goal & 36 \\
yds_togo & 11 & is_last_qb_kneel & 37 \\
yrds100 & 12 & is_last_os_kick & 38 \\
\hline
\end{tabular}

\section{Discussion}

The hypothesis motivating this study was that turnovers in NFL professional football are predictable. Statistical models were trained to predict the likelihood of observing a turnover on a given play from scrimmage. Empirical data representing seven complete NFL seasons (2009-2015) were used to train, test and evaluate the models.

Our machine learning results suggest evidence to support the hypothesis. Under certain conditions, both fumbles and interceptions can be anticipated at low false discovery rates (less than $15 \%)$. This means that when a turnover is predicted on the impending play from scrimmage, a high degree of confidence can be associated with that prediction.

The operational premise from the defender's perspective is that the impending play type (Run, Pass, Run or Pass) can be reliably estimated, using statistics (e.g., [19]) or intuition, in advance of the ball snap. These three play type categories are the basis for data segmentation, and predictive models are developed for each in turn.

For coaching purposes, this approach may be useful during development of game plans, or to inform in-game strategies to mitigate the negative consequences of turnovers by an offensive team, or to maximize their probability by a defensive squad.

Turnovers occur for many reasons, both physical and mental. Interceptions are caused by many factors-tipped balls and errant passes miss their destination due to the pressure of a defensive pass rush; misreading the defensive scheme prior to the ball snap; excellent coverage downfield by defensive backs; lack of spatial awareness of players on the field. Fumbles can be produced by violent physical collisions; insufficient ball protection by the runner; defensive "stripping" of the ball during tackling; or quarterback sacks resulting in fumbles.

This investigation contributes to the sports science literature by demonstrating the predictability of in-game events often considered to be essentially random in their occurrence. To the author's 
knowledge, direct prediction of turnovers has not previously appeared in the literature, which has focused on retrospective statistical analyses of turnover margin in football games.

\subsection{Quantitative Results and Observations}

Two data samples were modeled to assess turnover predictability: an aggregate of all NFL teams, and 32 individual team samples. Seven full season-long records were used for all datasets. We define a "good" false discovery rate to be $F D R<0.15$. A positive prediction made by a model $(\hat{y}(\mathbf{x})=1)$ is correct at least $85 \%$ of the time.

The GBM models to predict turnovers generally are characterized by high positive predictive value, and moderate sensitivity.

Predictive results for the aggregated sample (Table 2) exhibit low false discovery rates (FDR) for each play type segment $(6.4 \%, 8.3 \%$, and $3.9 \%$ for Run or Pass, Pass and Run, respectively). These measures reflect outstanding positive predictive value in estimating turnover likelihood. Run plays displayed the greatest sensitivity, with $T P R=0.65 \%$.

Team-wise turnover predictions are summarized in Tables 3-5.

For the Run or Pass play segment, three-fifths of the team models (62.5\%) have FDR rates below the stated $15 \%$ goodness criterion. Sensitivities were moderate for all team models. This exemplifies the essential tradeoff between FDR and TPR in ROC space [14], and follows from the present objective of minimizing FDR for strategic utility of the method.

Pass play results showed that one-third of team models (32\%) had false discovery rates below the goodness threshold. TPR is in the $30 \%-40 \%$ range.

For Run plays, $22 \%$ of the team results displayed $F D R<0.15$. Sensitivity of the turnover predictions is good, as $69 \%$ of the models (22/32) had true positive rates greater than $60 \%$.

It is interesting to note that the lowest turnover prevalence by play type in the population (for Run plays) is associated with the highest degree of model sensitivity in out-of-sample predictions. The disparity in realized false positive rates between Tables 2 and 5 could, in part, be due to the absolute number of training observations available in the aggregate case versus the team-wise samples. Fewer examples in the latter may be insufficient to learn the joint distribution between input and output variables.

\subsection{Extreme Events Modeling and Sports}

Turnovers have a number of characteristics in common with "extreme" events produced by complex dynamical systems, which are inherently difficult to predict [6]. These attributes include the following:

(a) Low occurrence frequency. Previous estimates found an average $2.9 \%$ of passes by NFL quarterbacks were intercepted [4]; fumbles were turned over to the opposing team in $0.83 \%$ of all run and pass plays [20]. The present study covering years 2009-2015 shows average turnover rates of $2.4 \%$ and $0.6 \%$ for pass and run plays, respectively.

(b) Intermittency. Turnovers appear to be random events. In one study, nearly equal parts luck and talent were proposed to account to year-over-year variations in turnover differential for a given team [3]. Within a season, NFL turnovers correlate weakly with prior game performance . A team with a strong season-to-date record of winning the turnover battle is likely to regress to the mean; conversely, teams losing in turnover margin at a point within a season tend to improve on this statistic moving forward [5].

(c) Costliness. A positive turnover margin in a given game is a significant predictor correlated with winning that game [2]. Teams with a unit valued positive turnover margin with respect to the opponent win the game $70 \%$ of the time [3].

The methods developed here are informed by the nonlinear geophysical dynamics literature by framing the prediction as a classification task (e.g., [21]), using game state and its recent history as 
precursors to learn the turnover event. A related investigation from the sports scientific literature suggested the predictability of within-game events and associated outcomes using dynamical process modeling [22]. In that study, researchers found patterns of tempo and in-game scoring that were common to many different sports (American football, hockey, and basketball). They suggest that these cross-cutting themes may provide insight into psychological processes that affect teams' performance in a general manner.

\subsection{Standard Errors of Predictions}

The statistics used to evaluate predictive performance reflect the sampling distribution of sample means and their standard errors, averaged over numerous replicates, folds and trials. It is observed that the standard errors for $F D R$ are a significant fraction of the sample mean value. This holds true for most results obtained in this study. The standard error of the sample mean will decrease as $1 / \sqrt{n}$, where $n$ is the sample size, according to the Central Limit Theorem.

\subsection{Sampling Notes}

This study centered on macro-level game data, to test the hypothesis "turnovers are predictable" in NFL football. Our samples aggregated over teams, and over players for team-level predictive models. In extensions to this work, player-level data could be included to build individual player models; such models may provide additional insights beyond descriptive statistical summaries of turnovers in current usage.

Turnovers related to sacks were excluded from consideration, mainly due to their negligible sample size relative to run and pass plays. Only $5.1 \%$ of sacks produce turnovers.

Author Contributions: Joel R. Bock conceived and performed the numerical experiments, analyzed their results, and wrote the paper.

Conflicts of Interest: The author declares no conflict of interest.

\section{Appendix A. Model Training Algorithms}

The algorithm used to train and evaluate GBM models for NFL turnover prediction is sketched in the pseudo-code listing in Algorithm A1. This procedure was used for the aggregate sample (all teams, seasons 2009-2015). The scheme used for the teamwise samples is notionally similar. Details are described in Section 2.3.

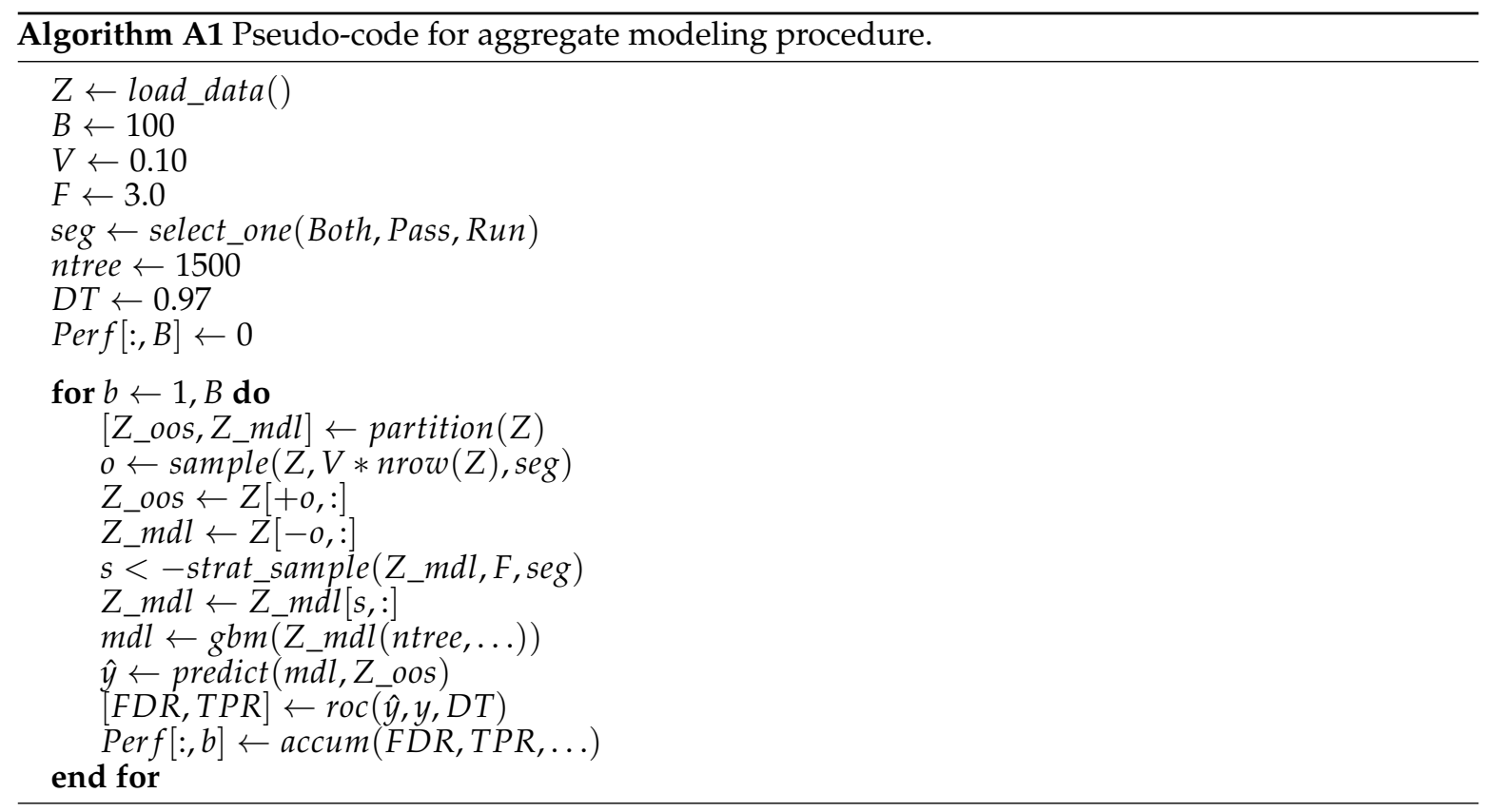




\section{References}

1. Goodell, R. Official Playing Rules of the National Football League; National Football League: New York, NY, USA, 2016.

2. Magel, R.C.; Childress, G. Examining the Outcome Effects of the Turnover Margin in Professional Football. Int. J. Sports Sci. Eng. 2012, 6, 147-152.

3. Ryan, J. Examining Luck in NFL Turnovers. Available online: http://harvardsportsanalysis.org/2014/10/ how-random-are-turnovers / (accessed on 28 September 2016).

4. Burke, B. How Random Are Interceptions? Available online: http:/ / archive.advancedfootballanalytics.com/ 2010/11/how-random-are-interceptions.html (accessed on 28 September 2016).

5. Beuoy, M. NFL Turnover Differential and the Point Spread. Available online: http://www.inpredictable.com/ 2012/02/nfl-turnover-differential-and-point.html (accessed on 10 October 2016).

6. Kantz, H.; Altmann, E.G.; Hallerberg, S.; Holstein, D.; Riegert, A. Dynamical interpretation of extreme events: Predictability and predictions. In Extreme Events in Nature and Society; AlbKantz, S.E.A., Ed.; Springer: Berlin/Heidelberg, Germany, 2006; pp. 69-93.

7. Friedman, J. Greedy Function Approximation: A Gradient Boosting Machine. Ann. Stat. 2001, 29, 1189-1232.

8. Hastie, T.; Tibshirani, R.; Friedman, J. The Elements of Statistical Learning, 10th ed.; Springer Series in Statistics; Springer: New York, NY, USA, 2013.

9. R Core Team. R: A Language and Environment for Statistical Computing; R Foundation for Statistical Computing: Vienna, Austria, 2013.

10. Greg Ridgeway. GBM: Generalized Boosted Regression Models; R Package Version 2.1.1, 2015. Available online: https:/ /CRAN.R-project.org/package=gbm (accessed on 28 December 2016).

11. Horowitz, M. nflscrapR: R Package for Scraping NFL Data off Their JSON API. Available online: https://github.com/maksimhorowitz/nflscrapR (accessed on 2 October 2016).

12. Provost, F. Machine learning from imbalanced data sets 101. In Proceedings of the AAAI-2000 Workshop on Imbalanced Data Sets, American Association for Artificial Intelligence, Austin, TX, USA, 31 July 2000; pp. 1-3.

13. Efron, B.; Tibshirani, R. Boostrap methods for standard errors, confidence intervals, and other measures of statistical accuracy. Stat. Sci. 1986, 1, 54-75.

14. Fawcett, T. An introduction to ROC analysis. Pattern Recognit. Lett. 2006, 27, 861-874.

15. Benjamini, Y.; Hochberg, Y. Controlling the false discovery rate: A practical and powerful approach to multiple testing. J. R. Stat. Soc. Ser. B 1995, 57, 289-300.

16. Bleakley, K.; Biau, G.; Vert, J. Supervised reconstruction of biological networks with local models. Bioinformatics 2007, 23, i57-i65.

17. Caruso, F.; Kantz, H. Prediction of extreme events in the OFC model on a small world network. Eur. Phys. J. B 2011, 79, 7-11.

18. Breiman, L. Bagging Predictors. Mach. Learn. 1996, 24, 123-140.

19. Goldberg, M.; Gilfix, A.; Rachesky, S.; Steeg, N.V. Predicting Offensive Play-Calling in the NFL. Available online: http:/ / harvardsportsanalysis.org/2016/03/predicting-offensive-play-calling-in-the-nfl/ (accessed on 12 October 2016).

20. Burke, B. Fumble Rates by Play Type. Available online: http://archive.advancedfootballanalytics.com/ 2010/01/fumble-rates-by-play-type.html (accessed on 20 September 2016).

21. Hallerberg, S.; Bröcker, J.; Kantz, H. Prediction of Extreme Events; Lecture Notes in Earth Sciences, Chapter Nonlinear Time Series Analysis in the Geosciences; Springer: Berlin/Heidelberg, Germany, 2008; pp. 35-59.

22. Merritt, S.; Clauset, A. Scoring dynamics across professional team sports: Tempo, balance and predictability. EPJ Data Sci. 2014, 3, 4.

(C) 2016 by the author; licensee MDPI, Basel, Switzerland. This article is an open access article distributed under the terms and conditions of the Creative Commons Attribution (CC-BY) license (http:/ / creativecommons.org/licenses/by/4.0/). 